\title{
Feedback-Detection Strategies for Adaptive Modulation Systems
}

\author{
Anthony E. Ekpenyong, Student Member, IEEE, and Yih-Fang Huang, Fellow, IEEE
}

\begin{abstract}
This letter analyzes the performance of an adaptive modulation system, taking into account additive noise and fading on the feedback channel. It is shown that these feedback channel imperfections could significantly degrade the throughput gains of adaptive modulation over nonadaptive transmission. Specifically, feedback errors can result in an outage region in the low signal-tonoise ratio region. Two feedback receivers are proposed: one is based on the finite-state Markov channel model; and the other is a generalized Bayesian receiver. These receivers reduce the outage region due to feedback errors, and they can complement or be used as alternatives to error-control coding schemes.
\end{abstract}

Index Terms-Adaptive modulation, feedback communication, finite-state Markov channels (FSMCs), signal detection.

\section{INTRODUCTION}

A DAPTIVE transmission techniques optimize the spectral efficiency of a communication system by varying transmission parameters in consonance with the channel realization. Given perfect channel state information (CSI) at the transmitter and receiver, a significant gain in throughput is achievable, compared with nonadaptive schemes [1]. However, the CSI knowledge is usually imperfect due to noisy feedback channels, channel-estimation error at the receiver, and feedback delays in closed-loop adaptive transmission systems. While channel estimation and delay issues have been addressed in [2]-[6], it is generally assumed that the feedback channel is error-free for frequency-division duplexing (FDD) systems. This is justified by assuming that a sufficiently powerful error-control code can be employed. Practically, coding is done at the expense of bandwidth for transmitting useful information. Moreover, more powerful codes generally imply a larger decoding (or feedback) delay. Hence, it is beneficial to design systems that can deal with imperfect feedback channels.

This letter analyzes the impact of noisy feedback channels on the performance of a FDD adaptive modulation system (AMS), where the adaptation is based on short-term or small-scale fading. Prior work in this area includes the adaptive trellis-coded quadrature amplitude modulation (QAM) system of [7], where the feedback information is protected by

Paper approved by A. Zanella, the Editor for Wireless Systems of the IEEE Communications Society. Manuscript received February 16, 2005; revised August 18, 2005 and December 4, 2005. This work was supported in part by the Indiana 21st Century Fund for Research and Technology under Contract 6511077-01, in part by the U.S. Army under Contract DAAD 16-02-C-0057-P1, in part by the National Science Foundation under Grant EC02-03366, and in part by the Center for Applied Mathematics, University of Notre Dame. This paper was presented in part at the IEEE International Conference on Acoustics, Speech, and Signal Processing, Philadelphia, PA, March 2005.

A. E. Ekpenyong was with the Department of Electrical Engineering, University of Notre Dame, Notre Dame, IN 46556 USA. He is now with the San Diego Wireless Center, Texas Instruments, San Diego, CA 92121 USA (e-mail: aekpenyong@ti.com).

Y.-F. Huang is with the Department of Electrical Engineering, University of Notre Dame, Notre Dame, IN 46556 USA (e-mail: huang@nd.edu).

Digital Object Identifier 10.1109/TCOMM.2006.881352 an error-control code. The effect of arbitrary bit errors on a two-state (ON-OFF) system was shown in [8], where feedback power control was used to ensure a constant received power at the transmitter. In this letter, we generalize the work of [8] to an $N$-state $(N>2)$ AMS without feedback power control. The reverse (feedback) channel is characterized by an error-probability matrix, which depends on the feedback-detection scheme employed. By optimizing the switching thresholds based on an average bit-error rate (BER) constraint, it is shown that there exists an outage region at low signal-to-noise ratio (SNR), where adaptive modulation is not feasible. The impact of feedback error is also shown to be different from the degradation caused by outdated CSI [3].

We analyze the performance of various feedback-detection schemes, including maximum-likelihood (ML) and maximum a posteriori (MAP) receivers. Since the average received SNR for a slowly fading channel depends on the shadowing environment, it can be assumed to be constant with respect to the short-term fading for which the AMS is designed. Therefore, it is realistic to assume reliable feedback of the average SNR, and it is shown that the feedback system can be formulated as a classical multiple-hypotheses testing problem. Conventional wisdom states that the average feedback BER for a MAP receiver is upper-bounded by the average feedback BER for the ML receiver. However, this performance advantage does not translate to an improved performance for adaptive modulation on the forward channel. In fact, it would be shown that this is only true for low-to-moderate average SNR. We also show how the MAP receiver can be generalized to a Bayesian receiver by defining a suitable cost function which favors conservative transmission because of the feedback error. The finitestate Markov channel (FSMC) model [9] for a slowly fading Rayleigh channel is also employed to design feedback receivers. We show that the FSMC-based receiver improves the performance of ML detection by exploiting the one-step transition property of the FSMC model.

A brief description of the AMS system is given in Section II. The system performance, given feedback error and delay, is analyzed in Section III, while the feedback-detection schemes are presented in Section IV. Performance results are shown in Section V, and we summarize our findings in Section VI. Throughout the letter, the superscripts ${ }^{f}$ and ${ }^{r}$ denote, respectively, the forward and reverse channels.

\section{SYSTEM DESCRIPTION}

Consider an AMS with $N$ QAM constellations, $\mathcal{M}=$ $\left\{M_{i}\right\}_{i=0}^{N-1}$, where $M_{0}$ denotes the no-transmission mode. We assume a statistically stationary time-selective flat-fading Rayleigh channel characterized by the Doppler frequency, $f_{d}$. To focus on feedback-channel imperfections, perfect CSI is assumed at the receiver, though some feedback delay $\tau$ is 
allowed. The symbol-sampled equivalent complex baseband model for the $k$ th received symbol is

$$
y(k)=\sqrt{\mathcal{P}^{f}} h^{f}(k) x(k)+z^{f}(k)
$$

where $h^{f}(k) \sim \mathcal{C N}(0,1)$ is the channel coefficient, and $z^{f} \sim \mathcal{C N}(0,1)$ is the additive complex Gaussian noise. The transmit power is denoted by $\mathcal{P}^{f}$, while $x(k)$ is an $M$-ary QAM (M-QAM) data symbol from $\mathcal{M}$ with unity average energy. Thus, the received instantaneous SNR is $\gamma(k)=\left|h^{f}\right|^{2} \mathcal{P}^{f}$, and the average SNR $\bar{\gamma}=\mathcal{P}^{f}$. A delayed CSI estimate $\hat{\gamma}$ is quantized and sent to the transmitter for adaptive transmission. Let $\mathcal{S}=\left\{S_{0}, S_{1}, \ldots, S_{N-1}\right\}$ denote the CSI state-space, where $S_{i}=\left\{\hat{\gamma}: y_{i} \leq \hat{\gamma}<y_{i+1}\right\}$, and the threshold vector $\mathbf{y}=\left[y_{1}, \ldots, y_{N-1}\right]\left(y_{0}=0\right)$ is to be optimized given an

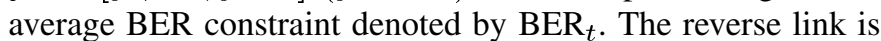
characterized by an error-probability matrix $Q=\left[q_{i, j}\right], 0 \leq i$, $j \leq N-1$, where $q_{i, j}$ is the probability that $M_{i}$ is selected for transmission when $S_{j}$ is the true state. The exact value of $Q$ depends on the feedback-detection scheme employed, and will be explored in detail in Section IV. In this manner, the effect of feedback error on system performance can be studied for any given error-probability matrix $Q$, as we show in the next section.

\section{FEEDBACK-ERror ANALYSIS}

In this section, we derive expressions for the spectral efficiency and average BER given a delayed and quantized channel realization which is also impacted by errors on the reverse link. Let $F_{\hat{\gamma}}(y), f_{\hat{\gamma}}(y)$ denote, respectively, the cumulative distribution function (cdf) and probability density function (pdf) for the delayed CSI estimate. The $i$ th transmission probability is obtained by averaging over all possible feedback decision events as

$$
\pi_{i}^{\prime}=\sum_{j=0}^{N-1} \pi_{j} q_{i, j}, \quad i=0, \cdots, N-1
$$

where $\pi_{j}=F_{\hat{\gamma}}\left(y_{j+1}\right)-F_{\hat{\gamma}}\left(y_{j}\right)$ is the $j$ th state probability. Thus, for discrete rate transmission, the spectral efficiency is given by

$$
\mathrm{SE}=\sum_{i=1}^{N-1} R_{i} \pi_{i}^{\prime}
$$

where $R_{i}=\log _{2} M_{i}$. In adaptive modulation, it is convenient to use approximate BER expressions for QAM [or phase-shift keying (PSK)] transmission over additive white Gaussian noise (AWGN) channels. Let $P_{b}\left(e \mid \gamma, \hat{\gamma}, M_{i}\right)=0.2 e^{-g_{i} \gamma}$ denote the instantaneous BER expression for the $i$ th constellation, where $\left\{g_{i}\right\}_{i=1}^{N-1}$ are modulation-specific constants (see, e.g., [4]). For simplicity, $\hat{\gamma}$ is normalized by the average SNR $\bar{\gamma}$ throughout the letter. By averaging $P_{b}\left(e \mid \gamma, \hat{\gamma}, M_{i}\right)$ over $\gamma$, it can be shown that $[2$, eq. 50$]$

$$
P_{b}\left(e \mid \hat{\gamma}, M_{i}\right)=c_{1, i} e^{-c_{2, i} \hat{\gamma}}
$$

where $c_{1, i}=0.2 /\left(1+g_{i}(1-\rho) \bar{\gamma}\right), c_{2, i}=\left(g_{i} \rho \bar{\gamma}\right) /\left(1+g_{i}(1-\right.$ $\rho) \bar{\gamma})$, and $\rho=J_{0}^{2}\left(2 \pi f_{d} \tau\right)$ is the cross-correlation coefficient between $\gamma$ and $\hat{\gamma} . J_{0}(\cdot)$ is the zeroth-order Bessel function of the first kind. Considering the feedback error, the average BER is given by

$$
\overline{\mathrm{BER}}=\frac{\mathrm{BER}^{\prime}}{\mathrm{SE}}=\frac{\sum_{i=1}^{N-1} R_{i} \sum_{j=0}^{N-1} q_{i, j} \mathrm{BER}_{i, j}}{\mathrm{SE}}
$$

where $\mathrm{BER}_{i, j}=\int_{S_{j}} P_{b}\left(e \mid y, M_{i}\right) f_{\hat{\gamma}}(y) d y$. An exact instantaneous BER expression can also be used: namely, [10]

$$
P_{b}\left(e \mid \hat{\gamma}, M_{i}\right)=\sum_{l=1}^{L} A_{i, l} \operatorname{erfc}\left(\sqrt{a_{i, l} \hat{\gamma}}\right), \quad i=1, \ldots, N-1
$$

where $\operatorname{erfc}(\cdot)$ is the complementary error function, and the number of terms $L$ depends on the QAM constellation. For simplicity, we only use the first two terms for all QAM constellations, where $\left[a_{i, 1}, a_{i, 2}\right]=\left[g_{i}, 9 g_{i}\right]$ and

$$
A_{i, l}= \begin{cases}\frac{\sqrt{M_{i}}-l}{\sqrt{M_{i} \log _{2} \sqrt{M_{i}}},} & \text { square M-QAM } \\ \frac{1}{\log _{2} M_{i}}\left(\frac{I-l}{I}+\frac{J-l}{J}\right), & \text { rectangular M-QAM }\end{cases}
$$

for $l=1,2$ and $I \cdot J=M_{i}$ for rectangular M-QAM. Thus, defining $b_{i, l}=1+a_{i, l}$, we have that $\mathrm{BER}_{i, j}=$ $\sum_{l=1}^{L} D\left(l, S_{j}, M_{i}, \bar{\gamma}\right)$, where

$$
\begin{aligned}
& D\left(l, S_{j}, M_{i}\right) \\
& =\int_{y_{j}}^{y_{j+1}} A_{i, l} \operatorname{erfc}\left(\sqrt{a_{i, l} y}\right) e^{-y} d y \\
& =A_{i, l}\left\{\left(e^{-y_{j}} \operatorname{erfc}\left(\sqrt{a_{i, l} y_{j}}\right)-e^{-y_{j+1}} \operatorname{erfc}\left(\sqrt{a_{i, l} y_{j+1}}\right)\right)\right. \\
& \quad-\left(a_{i, l} / b_{i, l}\right)^{1 / 2} \\
& \left.\quad \times\left(\operatorname{erfc}\left(\sqrt{b_{i, l} y_{j}}\right)-\operatorname{erfc}\left(\sqrt{b_{i, l} y_{j+1}}\right)\right)\right\} .
\end{aligned}
$$

\section{A. Threshold Optimization}

To compute the switching thresholds, an average BER constraint (instead of the instantaneous BER constraint employed in [11]) is used in this letter. One advantage of this approach is that by fixing the average BER to be no greater than $\mathrm{BER}_{t}$, the spectral efficiency is sufficient to quantify the impact of feedback errors. The optimization problem can be stated as

$$
\max _{\mathbf{y}} \operatorname{SE}(\mathbf{y}), \quad \text { subject to } C(\mathbf{y}) \leq 0
$$

where the constraint function $C(\mathbf{y})=\mathrm{BER}^{\prime}-\mathrm{SE} \cdot \mathrm{BER}_{t}$, and $\mathrm{BER}^{\prime}$ is the numerator of (5). Using the Lagrange multiplier $\lambda$, the unconstrained optimization problem is [12]

$$
J(\mathbf{y})=\left(1-\lambda \mathrm{BER}_{t}\right) \mathrm{SE}(\mathbf{y})+\operatorname{BER}^{\prime}(\mathbf{y}) .
$$

Solving the gradient function $\nabla_{\mathbf{y}} J=0$ it can be shown that

$$
\begin{aligned}
\Psi\left(y_{i}\right) & \triangleq \frac{\sum_{j=1}^{N-1} P_{b}\left(e \mid y_{i}, M_{j}\right) R_{j}\left(q_{j, i}-q_{j, i-1}\right)}{\sum_{j=1}^{N-1} R_{j}\left(q_{j, i}-q_{j, i-1}\right)} \\
& =\mathrm{BER}_{t}-\lambda^{-1}
\end{aligned}
$$




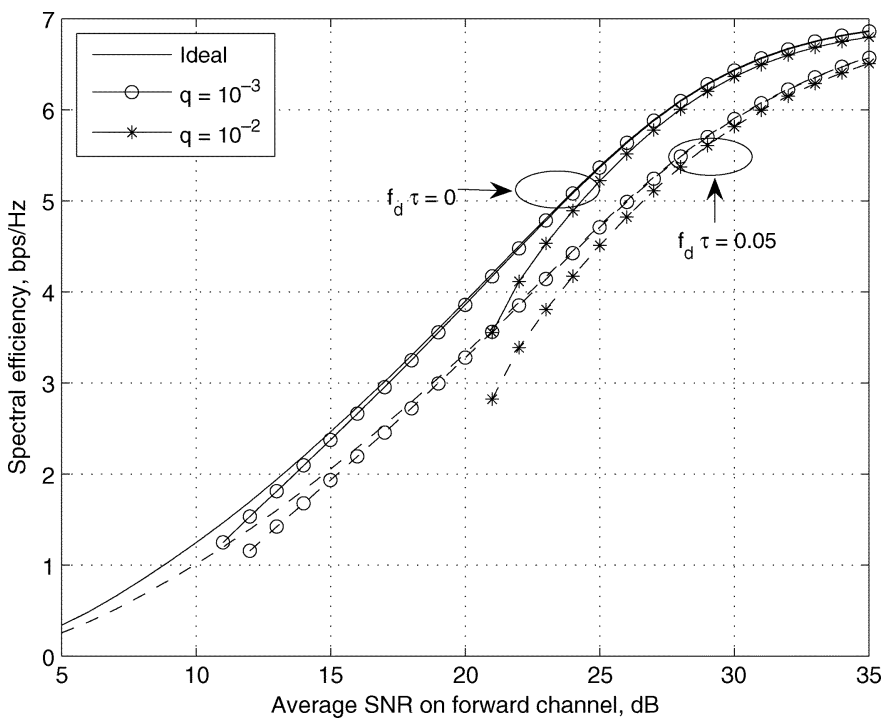

Fig. 1. Spectral efficiency for an AMS accounting for feedback error and feedback delay.

for $i=1, \ldots, N-1$. Therefore, selecting $y_{1} \in[0, \infty)$ and substituting in (11), we can obtain $y_{j}\left(y_{1}\right)$ by setting $\Psi\left(y_{j}\right)=$ $\Psi\left(y_{1}\right), j=2, \ldots, N-1$ to satisfy (11). The optimal threshold vector is then the vector $\mathbf{y}\left(y_{1}\right)$ that simultaneously satisfies the constraint and maximizes the spectral efficiency. A similar optimization technique was used in [12] for an AMS with an ideal feedback channel. The result here is different because the existence of a feasible region $\left\{y_{1}: C\left(\mathbf{y}\left(y_{1}\right)\right) \leq 0\right\}$ cannot be guaranteed due to feedback error. Note from (3) that for a fixed threshold vector $\mathbf{y}$, the spectral efficiency is relatively unaffected by feedback error due to averaging of the possible error events. However, this is not the case for the average BER, which can be shown by studying the limiting cases of low and high average SNR $\bar{\gamma}$. As $\bar{\gamma} \rightarrow 0, S_{0}$ is the most likely state, thus, transmission should be turned off. Feedback error would cause a higher constellation to be transmitted, which significantly increases the instantaneous BER. As a result, the average BER constraint may not be satisfied, implying that adaptive modulation is impractical in this region. Conversely, when $\bar{\gamma} \rightarrow \infty$, $S_{N-1}$ is the most likely state; as such, feedback error would not increase the average BER, but would reduce the spectral efficiency, since a lower constellation is erroneously transmitted. Nevertheless, it is important to note that for high $\bar{\gamma}$, a feasible $\mathbf{y}$ can be found to satisfy $C(\mathbf{y}) \leq 0$.

To illustrate the impact of feedback error combined with feedback delay, consider an AMS $\mathcal{M}=[0,2,4,8,16,32,64,128]$ and $\mathrm{BER}_{t}=10^{-3}$. Fig. 1 shows the spectral efficiency versus the average SNR on the forward channel (in steps of $1 \mathrm{~dB}$ ) for a specified reverse-channel BER of $q=10^{-3}$ and $q=10^{-2}$. The solid lines indicate a normalized delay $f_{d} \tau=0$, while the dashed lines are for $f_{d} \tau=0.05$. For $q=10^{-3}$, there is an outage (infeasible) region when $\bar{\gamma}<11 \mathrm{~dB}$. This outage region increases up to $21 \mathrm{~dB}$ for $q=10^{-2}$. The impact of feedback delay is seen to be an increased average SNR requirement (to achieve the same spectral efficiency), but the outage region is relatively unchanged. Thus, the effect of the feedback error is quite different from that of delay (the same trend can be seen if channel estimation/prediction error is taken into account). This is partly due to the fact that feedback errors occur on the quantized channel state, while delay (or estimation error) affects the unquantized (or analog) channel state. Therefore, in the next section, we consider only the feedback error and design receivers that will reduce the outage region.

\section{FeEdBack-Detection Schemes}

Given the performance expressions of the previous section, we now design feedback receivers to reduce the impact of feedback error. We begin by describing the reverse-link signal model. Let $\mathcal{U}=\left\{\mathbf{u}_{1}, \ldots, \mathbf{u}_{N-1}\right\}$ be a set of binary phase-shift keying (BPSK) symbol vectors of length $b\left(b=\log _{2} N\right)$, such that $\hat{\gamma} \in S_{i} \Rightarrow \mathbf{u}_{i}$ is transmitted. ${ }^{1}$ The average energy of each symbol is set to unity, thus the feedback signal model is given by

$$
\mathbf{v}(n)=\sqrt{\mathcal{P}^{r}} h^{r}(n) \mathbf{u}(n)+\mathbf{z}^{r}(n)
$$

where $\mathbf{u}(n) \in \mathcal{U}, h^{r} \sim \mathcal{C} \mathcal{N}(0,1)$ is the feedback channel realization, $\mathcal{P}^{r}$ is the reverse-link transmit power, and $\mathbf{z}^{r} \in$ $\mathcal{C N}\left(\mathbf{0}, \mathbf{I}_{b}\right)$ is the additive noise vector, where $\mathbf{I}_{b}$ is the $b \times b$ identity matrix. The time index $n$ is different from that used in (1), because the feedback rate is typically slower than the data rate [1]. For practical systems, $b$ is small so that $h^{r}$ is approximately constant for $b$ symbols. It is also assumed that the reverse-channel realization is known to the adaptive transmitter. Then, conditioned on $h^{r}$, a simple approximation for the error probability is that bit errors occur independently, i.e., each of the $b$ bits experiences a binary symmetric channel with crossover probability $q$. Define $d$ as the Hamming distance between $\mathbf{u}_{i}$ and $\mathbf{u}_{j}$. Then, the entries of the error-probability matrix $Q$ are computed as $q_{i, j}=\mathrm{E}_{h^{r}}\left\{q^{d}\left(1-q^{b-d}\right) \mid h^{r}\right\}$. We now consider Markov-based and Bayesian receivers that both exploit the statistical properties of the channel.

\section{A. Markov-Based Detection}

In this subsection, we show that ML feedback detection can be improved significantly by employing the FSMC model [9], which is known to be a good approximation to a slow timeselective Rayleigh fading channel. The FSMC model assumes that state transitions only occur to adjacent states, i.e.,

$$
P_{i, j} \triangleq \operatorname{Pr}\left(S(n)=S_{i} \mid S(n-1)=S_{j}\right)=0, \quad \text { for }|i-j|>1
$$

where $S(n)$ denotes the state at time $n$ and $P_{i, j}$ is the state-transition probability. For a normalized Doppler $f_{d} T_{s} \leq 10^{-3}$, where $T_{s}$ is the symbol duration, at most three events need to be characterized at each time instant, namely, a transition to the right (higher SNR region), one to the left (lower SNR region), or no change. As such, two approaches can be taken: 1) differential feedback - two bits are sufficient to fully describe all channel-state transitions; 2) full state feedback of $b$ bits-the feedback receiver can use this bounded state-transition property

\footnotetext{
${ }^{1}$ BPSK is chosen both for simplicity and for its comparatively high noise immunity in uncoded transmission.
} 
to correct some feedback errors. Due to space limitations, we only describe the full-state FSMC scheme here (see [11] for a description of the differential scheme).

Following the ML detection of the feedback signal in (12), the feedback detector can be substantially improved by limiting postdetection state transitions to adjacent states. If $S(n-1)=$ $S_{m}$, the feasibility set at time $n$ is $\mathcal{F}_{n}=\left\{S_{m-1}, S_{m}, S_{m+1}\right\}$ and $S(n) \in \mathcal{F}_{n}$ by the FSMC-bounded transition property. Hence, error propagation occurs if the postdetection feasibility set $\hat{\mathcal{F}}_{n} \neq \mathcal{F}_{n}$. For example, let $\hat{S}(n) \in \mathcal{F}_{n}$ but $\hat{S}(n) \neq S(n)$. Then, at the next time instant, an erroneous feasibility set $\hat{\mathcal{F}}_{n+1}$, which is based on $\hat{S}(n)$, would be used for detection, and this affects subsequent decisions. Therefore, to analyze the performance of this detector, we derive a lower bound to the $\overline{\mathrm{BER}}$ performance by assuming that $\mathbf{u}(n-1)$ is correctly received. Let $\mathbf{u}_{j} \rightarrow(\cdot, \ldots, \cdot)$ refer to the event that $\mathbf{u}_{j}$ is incorrectly decoded as an element of a subset of $\mathcal{U}$. Then, the detection events leading to transmission of $M_{i}$ may be enumerated as follows:

$$
\begin{array}{ll}
\left.\mathcal{E}_{1}\right) \quad\left\{S(n)=S_{i} \text { and no feedback error }\right\} ; \\
\left.\mathcal{E}_{2}\right) \quad\left\{S(n-1)=S_{i-1}, S(n)=S_{i-2}, \mathbf{u}_{i-2} \rightarrow\right. \\
& \left.\left(\mathbf{u}_{i}, \ldots, \mathbf{u}_{N-1}\right)\right\} ; \\
\left.\mathcal{E}_{3}\right) \quad\left\{S(n-1)=S_{i-1}, S(n)=S_{i-1}, \mathbf{u}_{i-1} \rightarrow\right. \\
& \left.\left(\mathbf{u}_{i}, \ldots, \mathbf{u}_{N-1}\right)\right\} ; \\
\left.\mathcal{E}_{4}\right) \quad\left\{S(n-1)=S_{i-1}, S(n)=S_{i}, \mathbf{u}_{i} \rightarrow\right. \\
& \left.\left(\mathbf{u}_{i+1}, \ldots, \mathbf{u}_{N-1}\right)\right\} ; \\
\left.\mathcal{E}_{5}\right) \quad\left\{S(n-1)=S_{i+1}, S(n)=S_{i}, \mathbf{u}_{i} \rightarrow\right. \\
\left.\quad\left(\mathbf{u}_{0}, \ldots, \mathbf{u}_{i-1}\right)\right\} ; \\
\left.\mathcal{E}_{6}\right) \quad\left\{S(n-1)=S_{i+1}, S(n)=S_{i+1}, \mathbf{u}_{i+1} \rightarrow\right. \\
\left.\quad\left(\mathbf{u}_{0}, \ldots, \mathbf{u}_{i}\right)\right\} ; \\
\left.\mathcal{E}_{7}\right) \quad\left\{S(n-1)=S_{i+1}, S(n)=S_{i+2}, \mathbf{u}_{i+2} \rightarrow\right. \\
\left.\quad\left(\mathbf{u}_{0}, \ldots, \mathbf{u}_{i}\right)\right\} ; \\
\left.\mathcal{E}_{8}\right) \quad\left\{S(n-1)=S_{i}, S(n)=S_{i-1}, \mathbf{u}_{i-1} \rightarrow \mathbf{u}_{i}\right\} ; \\
\left.\mathcal{E}_{9}\right) \quad\left\{S(n-1)=S_{i}, S(n)=S_{i+1}, \mathbf{u}_{i+1} \rightarrow \mathbf{u}_{i}\right\} .
\end{array}
$$

Notice that there is a reduction in the number of regions for which $M_{i}$ is erroneously transmitted to $S_{i-l}$, $|l| \leq 2, l \neq 0$. Each event is a product of independent forward and feedback channel events. For example, $\operatorname{Pr}\left\{\mathcal{E}_{5}\right\}=P_{i, i+1} \pi_{i+1} \sum_{m=0}^{i-1} q_{m, i}$. The state-transition probability can be approximated as [9]

$$
\begin{aligned}
P_{i-1, i} & =\operatorname{LCR}(i) T_{s} / \pi_{i}, \quad i=1, \ldots, N-1 \\
P_{i+1, i} & =\operatorname{LCR}(i+1) T_{s} / \pi_{i}, \quad i=0, \ldots, N-2
\end{aligned}
$$

where $\operatorname{LCR}(\cdot)$ is the level crossing rate, and for a normalized switching threshold $y_{j}$, it can be expressed as

$$
\operatorname{LCR}(j)=\sqrt{2 \pi y_{j}} f_{d} e^{-y_{j}}
$$

Note that $\left\{\mathcal{E}_{4}\right\}$ and $\left\{\mathcal{E}_{5}\right\}$ are the outcomes of ML decision errors which can be corrected by the FSMC receiver. These event probabilities can be inserted into (2), (3), and (5) to obtain the system performance. Apart from $\left\{\mathcal{E}_{1}\right\},\left\{\mathcal{E}_{4}\right\}$, and $\left\{\mathcal{E}_{5}\right\}$, the other events cause erroneous transmission, because in each case $S_{i} \in \mathcal{F}_{n}$.

A complementary method of exploiting the Markov model is useful when deep fades are taken into account. If there is a deep fade at time $n$, the feedback decision is discarded because it may be unreliable. In lieu of this feedback information, the worst-case state in $\mathcal{F}_{n}$, i.e., the lowest-rate QAM constellation in $\mathcal{F}_{n}$, is selected for transmission. This ensures that the target BER is always met though the spectral efficiency would be lower if the true channel state is actually higher than the worst case. This is similar in concept to the automatic repeat request (ARQ) feedback scheme of [13]. However, ARQ is not applicable for AMS feedback because of the delay issue. For the AMS, a decision must be made immediately on the feedback signal for transmitter adaptation.

\section{B. Bayesian Detection}

In most communication systems, transmitted symbols are assumed to be equally likely, thus, a minimum probability of error receiver is equivalent to an $\mathrm{ML}$ detector. However, this is not the case for the AMS feedback communication model, because feedback transmission depends on $\hat{\gamma}$. Since $\pi_{i}$ is functionally dependent on $\bar{\gamma}$, the set of state probabilities $\left\{\pi_{i}\right\}_{i=0}^{N-1}$ can be computed at the transmitter if $\bar{\gamma}$ is reliably known, as described in Section I. This implies that ML feedback detection may be suboptimal to MAP, or more generally, Bayesian detection. The decision error probabilities used in (2) and (5) can be computed by solving a classical multiple-hypotheses testing problem. This involves minimizing the Bayes risk [14]

$$
\bar{C}=\sum_{i=0}^{N-1} \sum_{j=0}^{N-1} C_{i j} \operatorname{Pr}\left(\mathcal{H}_{i} \mid \mathcal{H}_{j}\right) \pi_{j}
$$

where $\operatorname{Pr}\left(\mathcal{H}_{i} \mid \mathcal{H}_{j}\right)=q_{i, j}$ is the decision error event, and $C_{i j}$ is the cost of choosing $\mathcal{H}_{i}$ when $\mathcal{H}_{j}$ is true. An analytical computation of $\operatorname{Pr}\left(\mathcal{H}_{i} \mid \mathcal{H}_{j}\right)$ for the multiple-hypotheses problem is difficult, because the decision regions are determined by $(N-1)$-dimensional hyperplanes [14]. Therefore, in Section $\mathrm{V}$, the error probabilities are numerically computed. However, a closed-form solution exists for a two-state (binary hypothesis) system, and is presented here to compare the AMS performance for ML and MAP feedback receivers.

1) A Two-State System: Consider the two-state system $\left(\mathcal{H}_{0}, \mathcal{H}_{1}\right)$ of OFF-ON transmission, and let $C_{j j}=0$ for any $j$. Defining $\eta=\ln \left(C_{10} \pi_{0} /\left(C_{01} \pi_{1}\right)\right.$ and by noting that the transmitted sequences are real, the test statistic to determine the correct hypothesis is defined as

$$
\begin{aligned}
D(\mathbf{v}) \triangleq \Re\left\{\left(h^{r}\right)^{*}\left(\mathbf{u}_{1}-\mathbf{u}_{0}\right)^{T} \mathbf{v}\right\} & \\
& -\frac{1}{2}\left|h^{r}\right|^{2}\left(\left\|\mathbf{u}_{1}\right\|^{2}-\left\|\mathbf{u}_{0}\right\|^{2}\right) \underset{\mathcal{H}_{0}}{\stackrel{\mathcal{H}_{1}}{\gtrless}} \frac{1}{2} \eta,
\end{aligned}
$$

where $\Re\{\cdot\},{ }^{*}$, and ${ }^{T}$ denote, respectively, the real, conjugate, and transpose operations. Let $d_{1,0}$ be the Euclidean distance between $\mathbf{u}_{1}$ and $\mathbf{u}_{0}$. It can be shown [15] that conditioned on either hypothesis, $D(\mathbf{v})$ is Gaussian distributed with common variance 


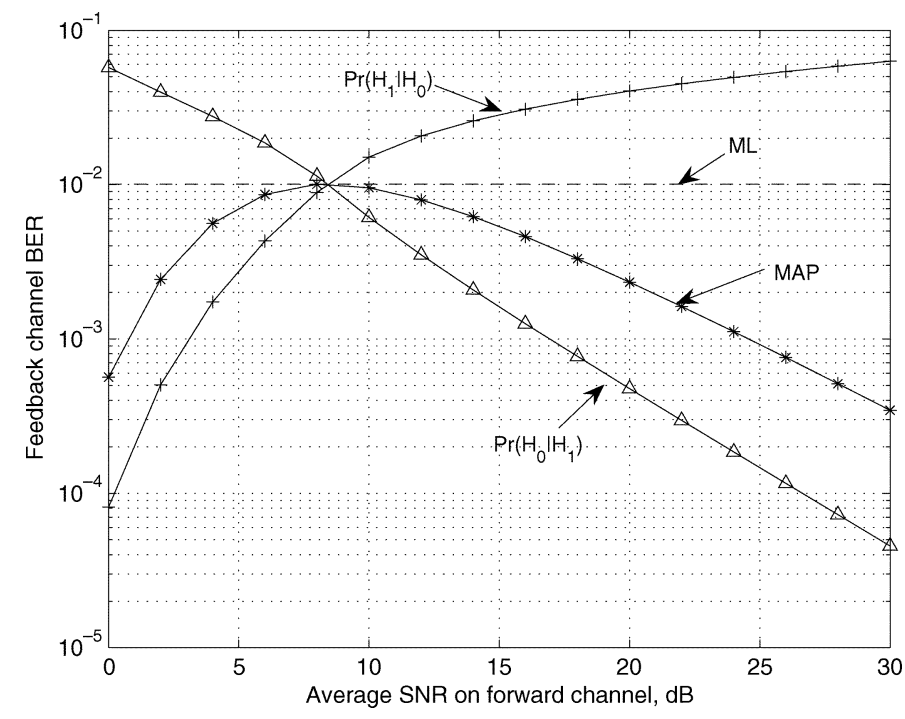

Fig. 2. Average feedback BER of a two-state BPSK system for ML and MAP feedback detection $(q=0.01)$.

$1 / 2\left|h^{r}\right|^{2} d_{1,0}^{2} \mathcal{P}^{r}$ and means $\pm 1 / 2\left|h^{r}\right|^{2} d_{1,0}^{2} \mathcal{P}^{r}$ for $\mathcal{H}_{1}$ and $\mathcal{H}_{0}$, respectively. Therefore, it is straightforward to compute the decision error probabilities as

$$
\operatorname{Pr}\left(\left.\mathcal{H}_{0}\left|\mathcal{H}_{1},\right| h^{r}\right|^{2}\right)=\frac{1}{2} \operatorname{erfc}\left(\sqrt{\frac{\left(d_{1,0}^{2}\left|h^{r}\right|^{2}-\eta\right)^{2}}{2 d_{1,0}^{2}\left|h^{r}\right|^{2}}}\right)
$$

and

$$
\operatorname{Pr}\left(\left.\mathcal{H}_{1}\left|\mathcal{H}_{0},\right| h^{r}\right|^{2}\right)=\frac{1}{2} \operatorname{erfc}\left(\sqrt{\frac{\left(d_{1,0}^{2}\left|h^{r}\right|^{2}+\eta\right)^{2}}{2 d_{1,0}^{2}\left|h^{r}\right|^{2}}}\right) .
$$

These expressions can then be used to obtain $q_{0,1}$ and $q_{1,0}$ by averaging over $\left|h^{r}\right|^{2}$. Note that (16) and (17) are generalized pairwise error probabilities for any pair of hypotheses $\left(\mathcal{H}_{i}, \mathcal{H}_{j}\right)$, $i, j \in[0, N-1]$. The special case of $\eta=0$ when $\pi_{0}$ and $\pi_{1}$ are assumed to be equally likely yields the well-known pairwise error-probability expression.

To analyze the system performance, let $C_{01}=C_{10}$, for simplicity, so that $\eta=\ln \left(\pi_{0} / \pi_{1}\right)$. Fig. 2 shows the average BER on the feedback channel for ML (dashed line), and MAP detection (starred line), which is a weighted sum of $\operatorname{Pr}\left(\mathcal{H}_{0} \mid \mathcal{H}_{1}\right)$ and $\operatorname{Pr}\left(\mathcal{H}_{1} \mid \mathcal{H}_{0}\right)$. BPSK is used for the ON state. If one considers the feedback channel as an independent system, then one can expect that MAP detection is better than ML detection, as seen in Fig. 2. Since we are dealing with a coupled system, this performance gain does not translate to the desired adaptive modulation performance on the forward channel. To see this, note that, in general, $\pi_{0} \neq \pi_{1}$. Thus, given (16) and (17), the adaptive transmitter's decision is biased in favor of the more likely hypothesis, since $\operatorname{erfc}(\cdot)$ is a decreasing function of its argument. For small $\bar{\gamma}$, where $S_{0}$ is the dominant state, $\eta>0$ and the inequality $\operatorname{Pr}\left(\mathcal{H}_{1} \mid \mathcal{H}_{0}\right)<\operatorname{Pr}\left(\mathcal{H}_{0} \mid \mathcal{H}_{1}\right)$ holds. This is desirable for the adaptive transmitter, because a wrong decision in favor of $\mathcal{H}_{1}$ increases the instantaneous BER. Conversely, at high $\bar{\gamma}$,

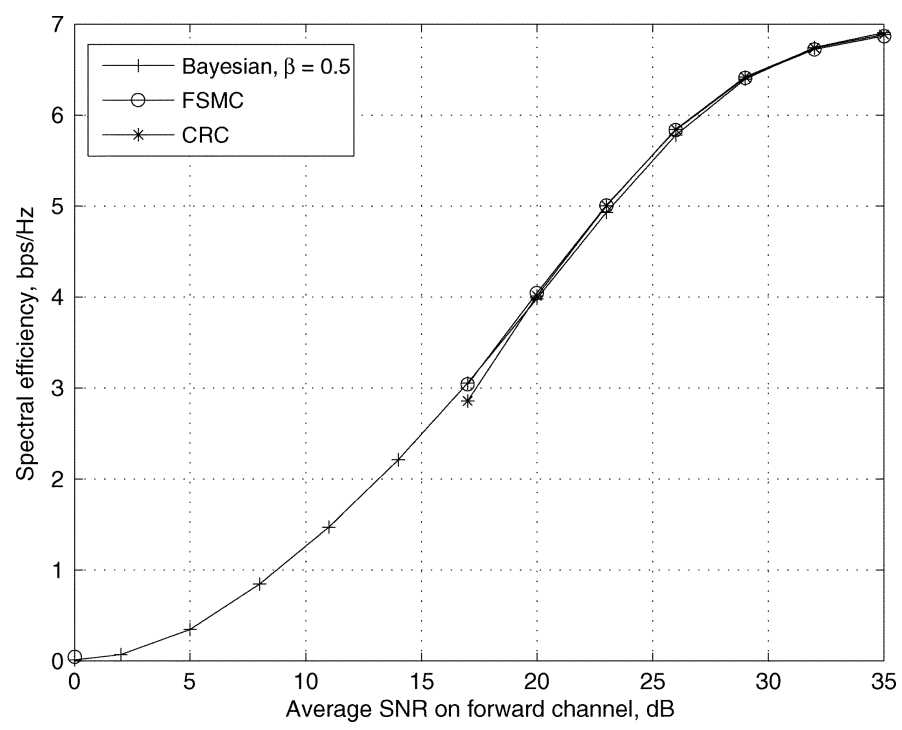

Fig. 3. Spectral efficiency for an AMS for different feedback detection schemes $\left(\mathrm{BER}_{t}=10^{-3}, f_{d} T_{s}=10^{-3}\right)$.

$\operatorname{Pr}\left(\mathcal{H}_{1} \mid \mathcal{H}_{0}\right)>\operatorname{Pr}\left(\mathcal{H}_{0} \mid \mathcal{H}_{1}\right)$, which leads to an increase in the average BER for the adaptive system due to erroneous transmission. These feedback-decision error probabilities determine the AMS performance on the forward channel, rather than the average BER of the feedback channel. Since the decision-error probabilities are equal for the ML receiver, it can be seen from Fig. 2 that ML may be preferable to MAP detection at high $\bar{\gamma}$. This result also holds for $N>2$, as would be confirmed in Section V.

While equal cost functions are common in traditional communication systems, a cost function can be defined for a qualitative metric of the effect of decision errors. In the AMS feedback case, it is desirable if $C_{i j}>C_{j i}$ and $C_{i j}>C_{k j}$ for $i>k>j$. Therefore, we propose the cost function

$$
C_{i j}=i-j, C_{j i}=\beta C_{i j}, \quad i>j, \beta \in(0,1] .
$$

In other words, the cost of transmitting at a higher rate than what the channel state calls for is greater than the cost of transmitting conservatively.

\section{RESULTS}

Numerical results are presented in this section for Bayesian and FSMC-based feedback receivers using the expressions in (6) and (8). For comparison, we also consider a cyclic redundancy check (CRC) error-detection scheme employing a $(7,3)$ Hamming code. In this scheme, when the feedback information is detected to be in error, it is discarded and the transmitted state is assumed to be the same as the state at the last adaptation instant. As in Fig. $1, N=8$ with the same QAM constellations, $\mathrm{BER}_{t}=10^{-3}$ and $f_{d} T_{s}=10^{-3}$. The error-probability matrix $Q$ is obtained by simulation for the Bayesian, FSMC, and CRC receivers, and is substituted in (9) to compute the optimal switching thresholds. The performance of these feedback schemes is shown in Fig. 3 for $q=0.01$ when using ML feedback detection. The Bayesian receiver with $\beta=0.5$ eliminates 


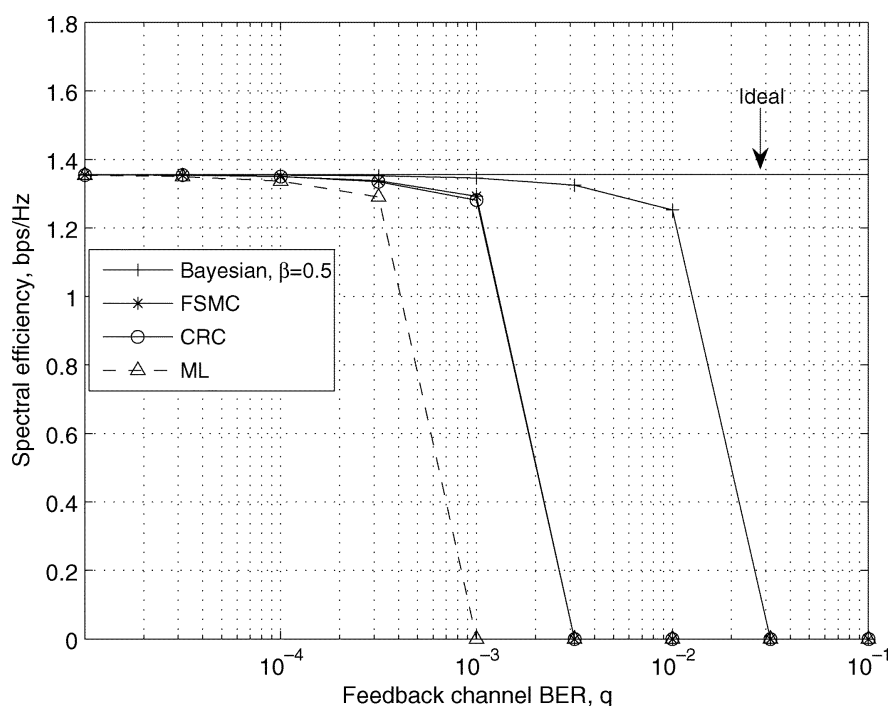

Fig. 4. Spectral efficiency versus feedback BER for different feedback-detection schemes and average forward channel SNR $\bar{\gamma}=10 \mathrm{~dB}\left(\mathrm{BER}_{t}=10^{-3}\right.$, $f_{d} T_{s}=10^{-3}$ ).

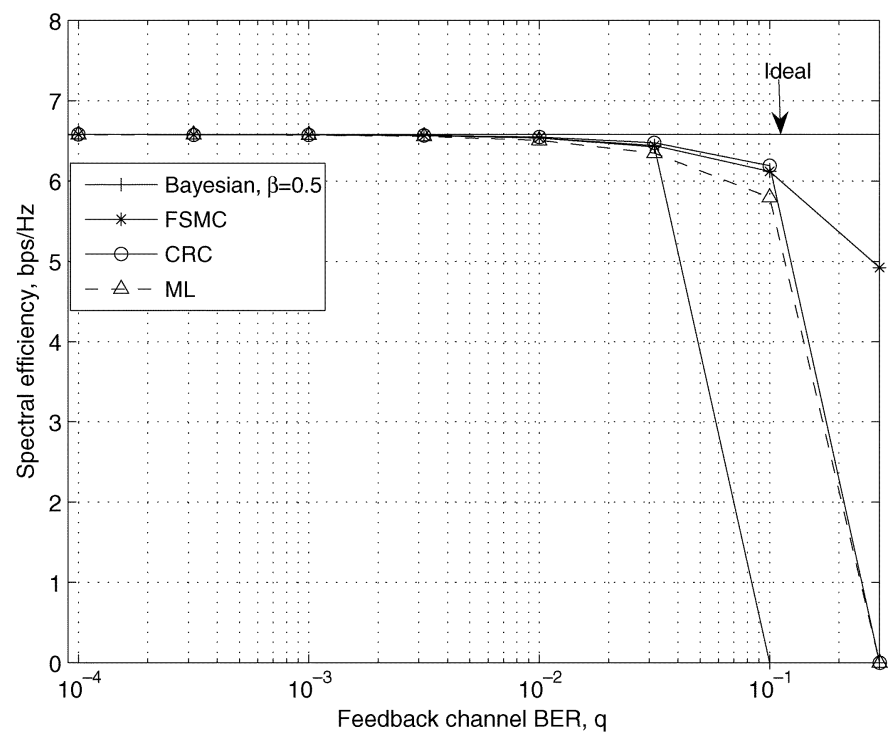

Fig. 5. Spectral efficiency versus feedback BER for different feedback-detection schemes and average forward channel SNR $\bar{\gamma}=30 \mathrm{~dB}\left(\mathrm{BER}_{t}=10^{-3}\right.$, $\left.f_{d} T_{s}=10^{-3}\right)$.

the outage region completely. The FSMC-based receiver compares well with the CRC receiver, and the outage region is reduced by $4 \mathrm{~dB}$, compared with the ML detection performance shown in Fig. 1.

The performance versus the feedback BER is shown in Figs. 4 $(\bar{\gamma}=10 \mathrm{~dB})$ and $5(\bar{\gamma}=30 \mathrm{~dB})$. The performance gain of the CRC and FSMC receivers over ML detection is evident in Fig. 4. It can also be seen that the Bayesian receiver has close-to-ideal performance for $q<0.01$. Interestingly, for a higher average SNR (Fig. 5), the FSMC and CRC receivers now have superior performance, compared with the Bayesian receiver. This shows that FSMC error-correction capability im- proves with $\bar{\gamma}$ partly because fewer decision errors are propagated. However, it should be recalled that the Bayesian receiver is not limited by the short-term fading rate, as is the FSMC receiver. It can also be seen that the ML receiver performance is better than the Bayesian receiver, which confirms the analysis in Section IV-B.1.

\section{CONCLUSION}

The impact of noisy feedback channels on the performance of an AMS has been investigated in this letter. It is shown that feedback error results in an outage region at low SNR, where adaptation is not feasible. Feedback-detection strategies, using prior statistical information, have been developed to mitigate the performance degradation caused by feedback error. The FSMCbased receiver exploits the Markov nature of the channel, while the Bayesian receiver exploits knowledge of the average SNR. It is shown that the outage region is reduced by employing these feedback receivers. An extension to this work can be to incorporate the impact of channel-estimation error of the forward channel at the receiver, and channel-estimation error of the reverse channel at the transmitter.

\section{REFERENCES}

[1] A. J. Goldsmith and S.-G. Chua, "Variable-rate variable-power MQAM for fading channels," IEEE Trans. Commun., vol. 45, no. 10, pp. 1218-1230, Oct. 1997.

[2] M.-S. Alouini and A. J. Goldsmith, "Adaptive modulation over Nakagami fading channels," Wireless Pers. Commun., vol. 13, no. 1, pp. 119-143, May 2000.

[3] D. L. Goeckel, "Adaptive coding for time-varying channels using outdated fading estimates," IEEE Trans. Commun., vol. 47, no. 6, pp. 844-855, Jun. 1999.

[4] S. Zhou and G. B. Giannakis, "How accurate channel prediction needs to be for transmit-beamforming with adaptive modulation over Rayleigh MIMO channels?," IEEE Trans. Wireless Commun., vol. 3, no. 4, pp. 1285-1294, Jul. 2004.

[5] G. Øien, H. Holm, and K. Hole, "Impact of channel prediction on adaptive coded modulation performance in Rayleigh fading," IEEE Trans. Veh. Technol., vol. 53, no. 3, pp. 758-769, May 2004.

[6] J. Paris, M. Aguayo-Torres, and J. Entrambasaguas, "Impact of channel estimation error on adaptive modulation performance in flat fading," IEEE Trans. Commun., vol. 52, no. 5, pp. 716-720, May 2004.

[7] V. K. N. Lau and M. D. Macleod, "Variable-rate adaptive trellis coded QAM for flat-fading channels," IEEE Trans. Commun., vol. 49, no. 9, pp. 1550-1560, Sep. 2001.

[8] J. Hämäläinen and R. Wichman, "Bit error probabilities in a two-rate communication system," in Proc. IEEE Int. Conf. Commun., Anchorage, AK, 2003, pp. 2693-2697.

[9] H. S. Wang and N. Moayeri, "Finite-state Markov channel—A useful model for radio communication channels," IEEE Trans. Veh. Technol., vol. 44, no. 1, pp. 163-171, Feb. 1995.

[10] K. Cho and D. Yoon, "On the general BER expression of one- and two-dimensional amplitude modulations," IEEE Trans. Commun., vol. 50, no. 7, pp. 1074-1080, Jul. 2002.

[11] A. E. Ekpenyong and Y.-F. Huang, "Markov channel-based feedback schemes for adaptive modulation systems," in Proc. IEEE Globecom, Dallas, TX, 2004, pp. 1091-1095.

[12] L. Hanzo, C. H. Wong, and M. S. Yee, Adaptive Wireless Transceivers. London, U.K.: Wiley, 2002.

[13] M. Zorzi and R. R. Rao, "Throughput analysis of ARQ selective-repeat protocol with time diversity in Markov channels," in Proc. IEEE Globecom, Singapore, 1995, pp. 1673-1677.

[14] H. L. Van Trees, Detection, Estimation, and Modulation Theory, Part 1. New York: Wiley, 1968.

[15] S. M. Kay, Fundamentals of Statistical Signal Processing, Volume 2-Detection Theory. Englewood Cliffs, NJ: Prentice-Hall, 1998. 